\title{
Anti-SEMA3A Antibody: A Novel Therapeutic Agent to Suppress Glioblastoma Tumor Growth
}

\author{
Jaehyun Lee, $B A^{1,2}$ \\ Yong Jae Shin, $P h D^{1,3}$ \\ Kyoungmin Lee, $\mathrm{BA}^{1,2}$ \\ Hee Jin Cho, $\mathrm{PhD}^{1}$ \\ Jason K. Sa, $P D^{1}$ \\ Sang-Yun Lee, $\mathrm{BA}^{1,2}$ \\ Seok-Hyung Kim, MD, PhD 1,24 \\ Jeongwu Lee, $\mathrm{PhD}^{5}$ \\ Yeup Yoon, $\mathrm{PhD}^{2}$ \\ Do-Hyun Nam, MD, PhD',23
}

${ }^{1}$ Institute for Refractory Cancer Research, Samsung Medical Center, Sungkyunkwan University School of Medicine, Seoul, ${ }^{2}$ Department of Health Sciences and Technology, Samsung Advanced Institute for Health Sciences and Technology (SAIHST),

Sungkyunkwan University, Seoul,

Departments of ${ }^{3}$ Neurosurgery and

${ }^{4}$ Pathology, Samsung Medical Center,

Sungkyunkwan University School of

Medicine, Seoul, Korea, ${ }^{5}$ Department of Stem

Cell Biology and Regenerative Medicine,

Lerner Research Institute, Cleveland Clinic,

Cleveland, $\mathrm{OH}, \mathrm{USA}$

Correspondence: Do-Hyun Nam, MD, PhD Department of Neurosurgery, Samsung Medical Center, Sungkyunkwan University School of Medicine and Department of Health Sciences and Technology, SAIHST, Sungkyunkwan University, 81 Irwon-ro, Gangnam-gu, Seoul 06351, Korea

Tel: 82-2-3410-3497

Fax: 82-2-3410-0048

E-mail: nsnam@skku.edu

Received June 29, 2017

Accepted October 30, 2017

Published Online November 10, 2017

\section{Purpose}

Glioblastoma (GBM) is classified as one of the most aggressive and lethal brain tumor. Great strides have been made in understanding the genomic and molecular underpinnings of GBM, which translated into development of new therapeutic approaches to combat such deadly disease. However, there are only few therapeutic agents that can effectively inhibit GBM invasion in a clinical framework. In an effort to address such challenges, we have generated anti-SEMA3A monoclonal antibody as a potential therapeutic antibody against GBM progression.

\section{Materials and Methods}

We employed public glioma datasets, Repository of Molecular Brain Neoplasia Data and The Cancer Genome Atlas, to analyze SEMA3A mRNA expression in human GBM specimens. We also evaluated for protein expression level of SEMA3A via tissue microarray (TMA) analysis. Cell migration and proliferation kinetics were assessed in various GBM patient-derived cells (PDCs) and U87-MG cell-line for SEMA3A antibody efficacy. GBM patient-derived xenograft (PDX) models were generated to evaluate tumor inhibitory effect of anti-SEMA3A antibody in vivo.

\section{Results}

By combining bioinformatics and TMA analysis, we discovered that SEMA3A is highly expressed in human GBM specimens compared to non-neoplastic tissues. We developed three different anti-SEMA3A antibodies, in fully human IgG form, through screening phagedisplayed synthetic antibody library using a classical panning method. Neutralization of SEMA3A significantly reduced migration and proliferation capabilities of PDCs and U87-MG cell line in vitro. In PDX models, treatment with anti-SEMA3A antibody exhibited notable tumor inhibitory effect through down-regulation of cellular proliferative kinetics and tumorassociated macrophages recruitment.

\section{Conclusion}

In present study, we demonstrated tumor inhibitory effect of SEMA3A antibody in GBM progression and present its potential relevance as a therapeutic agent in a clinical framework.

\author{
Key words \\ Glioblastoma, Semaphorin-3A, Neutralization, \\ Fully human antibody
}




\section{Introduction}

Glioma are categorized according to their grade based on histological and pathological features based on World Health Organization (WHO) classification. Glioblastoma (GBM, WHO grade IV glioma) is the most aggressive malignant primary brain tumor [1,2]. Current standard regimen consists of surgical resection followed by radiation and chemotherapy $[3,4]$. Despite such aggressive treatment option, the average survival of GBM patients spans only 1-2 years [4], requiring a new therapeutic approach.

Dynamic interaction between ligand and its partner receptor regulates essential cellular functions including cell survival, proliferation, and migration [5]. In cancerous tissues, including GBM, a multitude of ligand-receptor signaling axis are frequently dysregulated, resulting in tumor malignancy and dismal prognosis [6]. Recent large-scale genomic studies have revealed a catalog of core oncogenic pathways that are frequently activated in tumor progression including receptor tyrosine kinases that encompass epidermal growth factor receptor (EGFR), platelet-derived growth factor receptor, and c-MET $[7,8]$. As tumor cells often depend on oncogenic signaling pathways for survivability, inhibition of these pathways has been recognized as a potential therapeutic approach. Antibody-mediated therapy has been a promising approach, as shown by its clinical success involving antiEGFR antibodies [9]. Currently, 13 Food and Drug Administration approved antibodies have been translated into a clinical course and newly developed antibodies are currently being evaluated in various stages of clinical trials [10].

Semaphorins are able to regulate cell to cell interaction and differentiation [11]. There are 20 types of mammalian semaphorins which are categorized into five classes (classes 3-7) according to their molecular structures [12]. Among five classes, only class 3 Semaphorins are secreted proteins, whereas the rest are produced as membrane-anchored or transmembrane proteins [13]. Semaphorin3A (SEMA3A) is widely known as an axon guidance factor; however, recent studies have demonstrated its oncogenic roles in various cancer types [14-16]. In pancreatic cancer, SEMA3A promotes dissemination and invasion through activation of multiple pathways involving Rac1, GSK3, and p42 / p44 mitogen-activated protein kinase (MAPK) [17]. In addition, autocrine SEMA3A in GBM promotes cell dispersal by modulating substrate adhesion and elevates vascular permeability, resulting in tumor-promoted vascular leakage in vitro and in vivo $[18,19]$. SEMA3A in hepatocellular carcinoma functions as a chemoattractant involved in tumor-associated macrophages (TAM) infiltration and promotes tumor proliferation and migration [20]. These studies suggest SEMA3A as a potential therapeutic target in suppressing cancer prolifera- tion and invasion. However, development of SEMA3A targeting antibody has not been initiated. In present study, we generated fully human antibody that targets SEMA3A. Phage display technology has been widely employed for high-throughput generation of antibodies [21,22]. Through a single chain fragment variant (scFv) phage display screen, we isolated anti-SEMA3A scFvs. Using the Expi293F Expression System (Thermo Fisher Scientific, Waltham, MA), we established three different fully human anti-SEMA3A IgG antibodies and determined their therapeutic efficacy in GBM.

\section{Materials and Methods}

\section{Statistical analysis}

Expression profiling of Repository of Molecular Brain Neoplasia Data (REMBRANDT) glioma dataset were downloaded as CEL files. Expression levels of each sample were summarized per each gene using Affymetrix U133 Plus 2.0 array annotation file (Affymetrix, Santa Clara, CA). SEMA3A mRNA expression levels by WHO glioma grade were statistically compared via student $t$ test. For The Cancer Genome Atlas (TCGA) GBM data set, Kaplan-Meier survival analysis was available from cBioPortal (http:// www.cbioportal.org/).

\section{Screening of anti-SEMA3A scFv}

We performed selection of SEMA3A-binding phage scFv via classical panning method using synthetic human $\mathrm{scFv}$ phage library. The antigens that were used for panning were recombinant human SEMA3A (rhSEMA3A)/Fc chimeric protein (R\&D Systems, Minneapolis, MN) and Erbitux (Eli Lilly, Indianapolis, IN). Immunotubes (Nunc, Rochester, NY) were coated with $5 \mu \mathrm{g} / \mathrm{mL}$ of rhSEMA3A/Fc chimeric protein (R\&D Systems) in phosphate buffered saline (PBS) for 1 hour at $37^{\circ} \mathrm{C}$. After blocking the tube with $3 \%$ skim milk/ PBS, Erbitux was added and incubated for 1 hour in room temperature during the binding step to eliminate scFvs that specifically bind to the Fc portions of rhSEMA3A/Fc. Bound phage-scFvs were eluted from the tube by adding $1 \mathrm{~mL}$ of $100 \mathrm{mM}$ triethylamine and neutralized by adding $0.5 \mathrm{~mL}$ of 1.0 M Tris- $\mathrm{HCl}$ ( $\mathrm{pH}$ 7.4). For the rescue step, eluted phagescFvs were infected into $10 \mathrm{~mL}$ of Escherichia coli, ER2537 cells in Super Broth medium.

\section{Production and purification of anti-SEMA3A IgG}

The Expi293F expression system (Thermo Fisher Scientific) was used for anti-SEMA3A IgG production. Using HiTrap 
protein G HP (5 mL) of ÄKTA Protein Purification System (GE Healthcare, Barrington, IL) purified anti-SEMA3A antibodies were concentrated by Amicon Ultra Centrifugal Filter (Merck Millipore, Burlington, MA). The produced antiSEMA3A IgGs were evaluated by size exclusion high-performance liquid chromatography analysis and sodium dodecyl sulfate polyacrylamide gel electrophoresis (SDS-PAGE) for aggregation and degradation. In vivo experiment was further tested by LAL endotoxin quantitation kit (QCL-1000, Lonza, Basel, Switzerland).

\section{ELISA}

The flat bottom 96-well plates (Costar, Corning, New York, $\mathrm{NY}$ ) were coated with $1 \mu \mathrm{g} / \mathrm{mL}$ of human, mouse recombinant protein (R\&D Systems), Erbitux (IMC-C225), and bovine serum albumin (BSA; NEB, Ipswich, MA) overnight at $4^{\circ} \mathrm{C}$. Plates were washed with PBST $(0.1 \%)$ and blocked with 3\% skim milk (BD Difco, Franklin Lakes, NJ) solution. The anti-SEMA3A scFvs and IgGs were incubated for 1 hour in room temperature. After washing with PBST (0.1\%), antiHA antibody conjugated to horseradish peroxidase (HRP; $\mathrm{scFv}$ ) and the anti-human Fab antibody conjugated to HRP ( $\mathrm{IgG}$ ) were added for 1 hour in room temperature. Following washing step, each well was detected with tetramethylbenzidine (TMB) solution as HRP substrate for 5-25 minutes. The stop solution for TMB reaction was added and enzymelinked immunosorbent assay (ELISA) plate was analyzed at $450 \mathrm{~nm}$.

\section{Patient-derived GBM specimens}

GBM specimens were obtained from patients undergoing surgery based on consent in accordance with the appropriate Institutional Review Boards. Patient-derived GBM cells were cultured in the Neurobasal-A medium (NBA) [23].

\section{Proliferation assay}

Using a 96-well plate (Corning), GBM patient cells $\left(5 \times 10^{3}\right.$ cells / well) were seeded into $100 \mu \mathrm{L}$ of NBA medium and SEMA3A IgG or control human IgG (Thermo Fisher Scientific) were given for 3 days. After 6 and 9 days, $10 \mu \mathrm{L}$ of EZ-Cytox (Daeil Lab, Seoul, Korea) was added to the plates and incubated for 2 hours. The optical density was measured at $450 \mathrm{~nm}$ using a microplate reader (Bio-Rad, Hercules, CA). The cells were plated in quintuplicate.

\section{Migration assay}

Migration assays were performed using the Transwell system, in which the upper chambers of the transwells were coated with poly-L-ornithine for 1 hour and dried. $1 \times 10^{5}$ GBM cells in $100 \mu \mathrm{L}$ of media without any growth factors were seeded into the upper chambers and the lower chamber was filled with $600 \mu \mathrm{L}$ of medium including growth factors. The anti-SEMA3A antibodies (scFv, $50 \mu \mathrm{g} / \mathrm{mL} ; \mathrm{IgG}, 10 \mu \mathrm{g} /$ $\mathrm{mL}$ ) were added into the upper chambers and lower chambers and transwells were incubated for 24 hours at $37^{\circ} \mathrm{C}$. Next day, migrated cells on the lower surface of the upper chamber were fixed with methanol and stained with hematoxylin and eosin. The numbers of migrated cells were counted in eight different regions of the chambers that were selected randomly. The Oris cell migration assay (Platypus Technologies, Fitchburg, WI) was processed to evaluate antiSEMA3A antibody effect in a dose-dependent manner and U87-MG $\left(5 \times 10^{3}\right.$ cells / well) cells were seeded in a 96-well plate. After 24 hours, migrated cells were quantified with Calcein AM fluorescent dye stained.

\section{Subcutaneous xenograft tumor models}

Patient-derived cells (PDCs) were injected into the subcutaneous region of 6-week-old female BALB / c nude mice. The tumor bearing mice were regrouped when mass volume reached at average of $80 \mathrm{~mm}^{3}$. Anti-SEMA3A antibody F11 and PBS were administered via intravenous injection. Mice with body weight loss greater than $20 \%$ were sacrificed.

\section{Immunofluorescence analysis}

Subcutaneous tumors were fixed in 5\% paraformaldehyde and embedded in paraffin. Serial sections were stained with anti-SEMA3A (Abcam, Cambridge, MA) or anti-pERK (Cell Signaling Technology, Danvers, MA). Terminal deoxynucleotidyl transferase dUTP nick end labeling (TUNEL) assays were performed using the TUNEL apoptosis detection kit (Millipore, Billerica, MA). To evaluate TAM accumulation, paraffin sections were stained with anti-Iba1 (Abcam) as a macrophage marker.

\section{Ethical statement}

The study was approved by the Institutional Review Board of Samsung Medical Center (IRB No. 2010-04-004) and performed in accordance with the principles of the Declaration of Helsinki. Written informed consents were obtained.

All mouse experiments were performed according to the Association for Assessment and Accreditation of Laboratory Animal Care-accredited guidelines of our institute's Animal Use and Care Committee. 

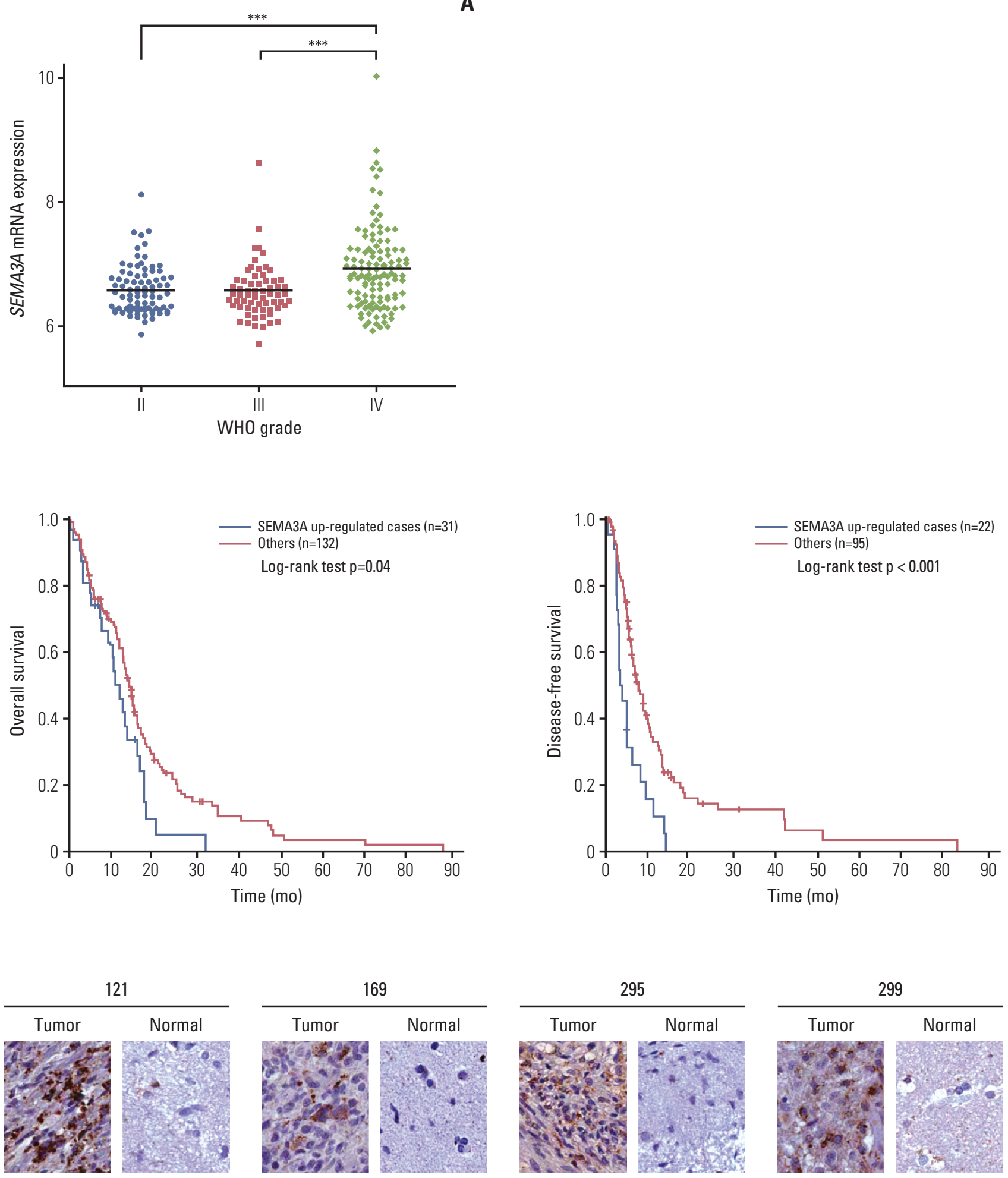

Fig. 1. SEMA3A is highly expressed in glioblastoma (GBM) specimens compared to the normal brains. (A) REMBRANDT microarray data analysis for SEMA3A mRNA expression levels corresponding to glioma grades II-IV. WHO, World Health Organization. ${ }^{* * *} \mathrm{p}<0.001$. (B) Kaplan-Meier survival curves of GBM patient corresponding to SEMA3A expression levels in The Cancer Genome Atlas datasets. (C) Immunohistochemistry of SEMA3A on tissue microarray. (Continued to the next page) 
SEMA3A expression in GBM patient paired samples ( $n=27$ )

\begin{tabular}{lcrrrr} 
& & \multicolumn{5}{c}{ Score } \\
\cline { 3 - 6 } Histology & No. & 0 & $1+$ & $2+$ & $3+$ \\
Normal & 27 & 7 & 17 & 3 & 0 \\
Tumor & 27 & 2 & 5 & 8 & 12 \\
\hline & & & & & $\mathrm{p}<0.001$
\end{tabular}

Fig. 1. (Continued from the previous page) (D) Representative analysis of SEMA3A expressions in 27 GBM paired tumor and normal specimens.

\section{Results}

\section{SEMA3A is highly expressed in GBM specimens com- pared to the normal brain tissues}

We first analyzed the mRNA expression levels of SEMA3A in gliomas according to their pathological grade (II to IV) using Rembrandt glioma dataset. SEMA3A expression was significantly correlated with the glioma grade level. GBM, in particular, exhibited higher expression levels of SEMA3A compared to grade II and III gliomas (Fig. 1A). Overall survival and progression-free survival of patients in TCGA showed that the SEMA3A high expression group portrayed worse survival rate $(p=0.04$ and $p<0.001)$ (Fig. 1B). Our results suggest that upregulation of SEMA3A expression is directly correlated with disease progression in GBM. To determine the SEMA3A protein level in GBM specimens, we conducted tissue microarray analysis on 27 GBM and matched adjacent non-neoplastic brain tissue samples. Immunohistochemical analysis showed elevated protein levels of SEMA3A in all GBM tissue samples, while the majority of non-tumor tissues showed minimal levels of SEMA3A (Fig. 1C and D). Collectively, the malignant effect of SEMA3A expression in GBM specimens is evident and SEMA3A appears to be an ideal therapeutic target in GBM treatment.

\section{Identification of SEMA3A-specific scFv using synthetic phage library}

In present study, we generated a $\mathrm{scFv}$ with high affinity to SEMA3A using a synthetic human scFv phage library. The synthetic human $\mathrm{scFv}$ phage library with a total diversity of $7.6 \times 10^{9} \mathrm{cfu}$ were composed of six diversified complementarity determining regions (CDRs) [24]. We performed a classical panning method using immunotube-immobilized rhSEMA3A/Fc chimeric protein. In order to prevent any
scFvs from binding to the Fc portion, we combined our synthetic scFv library with Erbitux containing the Fc region and proceeded on to the selection process. Approximately 70 -fold increase in output to input phage ratio was observed after the fourth selection round compared to the first, indicating the specificity of the accumulated phages to human SEMA3A (hSEMA3A) (Fig. 2A).

We incubated the final resulting outputs on LuriaBertani / ampicillin plate in $37^{\circ} \mathrm{C}$ overnight. Afterwards, we randomly picked 86 different single colonies and conducted phage ELISA. In accordance with the given criteria (at least two-fold increase to the negative control, i.e., BSA), 52 out of 86 colonies $(60 \%)$ were identified as rhSEMA3A binders (Fig. 2B). Erbitux was also used as a negative control (data not shown). Through sequence analysis of the 49 positive clones, we obtained three final clones that were specific for SEMA3A. Our final candidates, F11, A08, and C10, possessed specific sequences that are identical to other 22,4 , and 3 other clones, respectively. To produce purified forms of the generated $\mathrm{scFv}$, we inserted our three clones into E. coli Top10F, which recognizes the amber codon residing in the front of gene III and removes it in process. The resulting purified clones were verified through denaturation using Coomassie blue staining method (Fig. 2C). As the size of a typical scFv is at approximately $28 \mathrm{kDa}$, we expected our clones to appear between 25 and $37 \mathrm{kDa}$ markers. As we used PBS with 200 $\mathrm{mM}$ imidazole as our elution, eluted anti-SEMA3A scFv were processed under SDS-PAGE analysis without the imidazole. To examine the binding activity of the anti-SEMA3A scFvs, we measured the binding affinity of each clone through indirect ELISA analysis with at serial antibody concentration levels. We used as 12B clone as negative control scFv having no binding to human Sema3A (S1 Fig.). Our results showed that the binding activity of each antiSEMA3A clone to hSEMA3A protein was elevated in a dosedependent manner, while BSA, the control, displayed no significant changes (Fig. 2D). Overall, we have successfully produced three distinct scFvs that are specific to human SEMA3A using synthetic phage library.

\section{Production of anti-SEMA3A IgG antibodies}

To transform the anti-SEMA3A scFvs into their respective IgG forms, we transfected Expi293F cells with heavy and light chain regions of each anti-SEMA3A scFv. We purified three anti-SEMA3A IgG antibodies using the ÄKTA Protein Purification System and an Amicon Ultra Centrifugal Filter to produce A08, C10, and F11 clones at concentration levels of 118,138 , and $330 \mathrm{mg} / \mathrm{L}$, respectively. High performance liquid chromatography with a gel permeation chromatography column were used to validate the purity level of A08, C10, and F11 antibodies. Notably, all three clones exhibited 
A

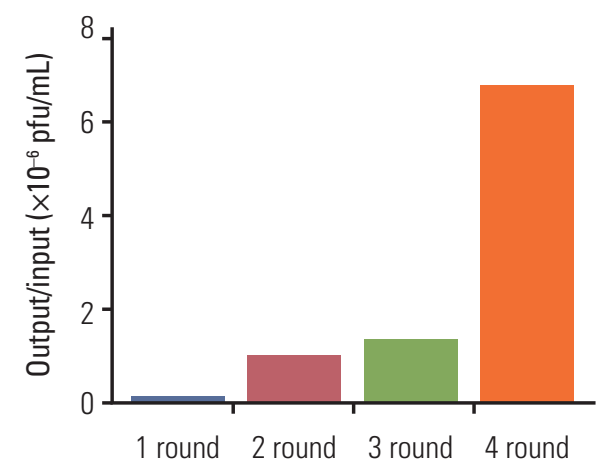

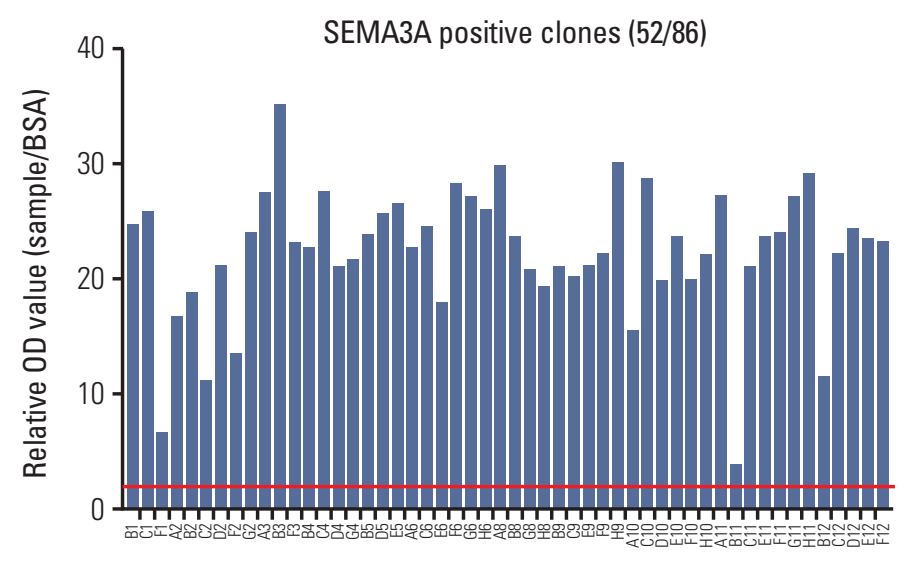
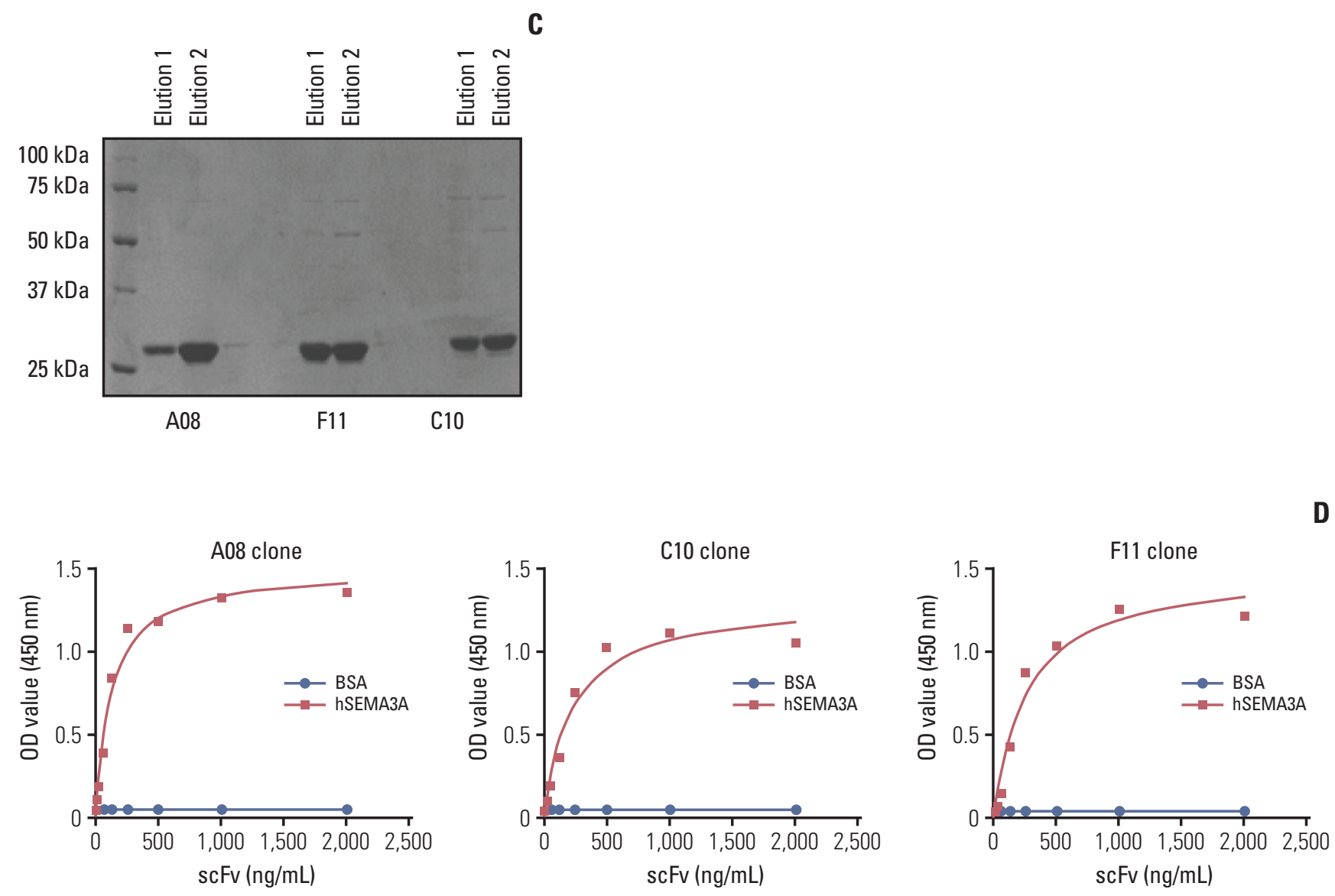

Fig. 2. Identification of SEMA3A-specific single chain fragment variant (scFv) using synthetic phage library. (A) The efficacy of classical panning was evaluated by the ratio of output phage titer to input phage titer. (B) Based on enzyme-linked immunosorbent assay (ELISA) results, 52 positive clones specific to human SEMA3A were selected from among 86 clones which were selected randomly in the fourth round of the output mixture. (C) The three scFvs purified using Ni-NTA resin and poly-column were eluted by phosphate buffered saline with $200 \mathrm{mM}$ imidazole (pH7.4). Molecular sizes of the three anti-SEMA3A clones (A08, C10, and F11) were verified by Coomassie blue staining. (D) hSEMA3A binding activities of three anti-SEMA antibodies and 12B (a negative control) were confirmed by indirect ELISA. 96-well plates coated with hSEMA3A $(1 \mu \mathrm{g} / \mathrm{mL}$ ) or bovine serum albumin (BSA; $1 \mu \mathrm{g} / \mathrm{mL}$ ) were bound for three anti-SEMA3A antibodies and 12B. Then, antihemaglutinin antibody conjugated to horseradish peroxidase (HRP) was used for bound scFvs detection. The absorbance of each well for HRP reactions was measured at $450 \mathrm{~nm}$. OD, optical density. 

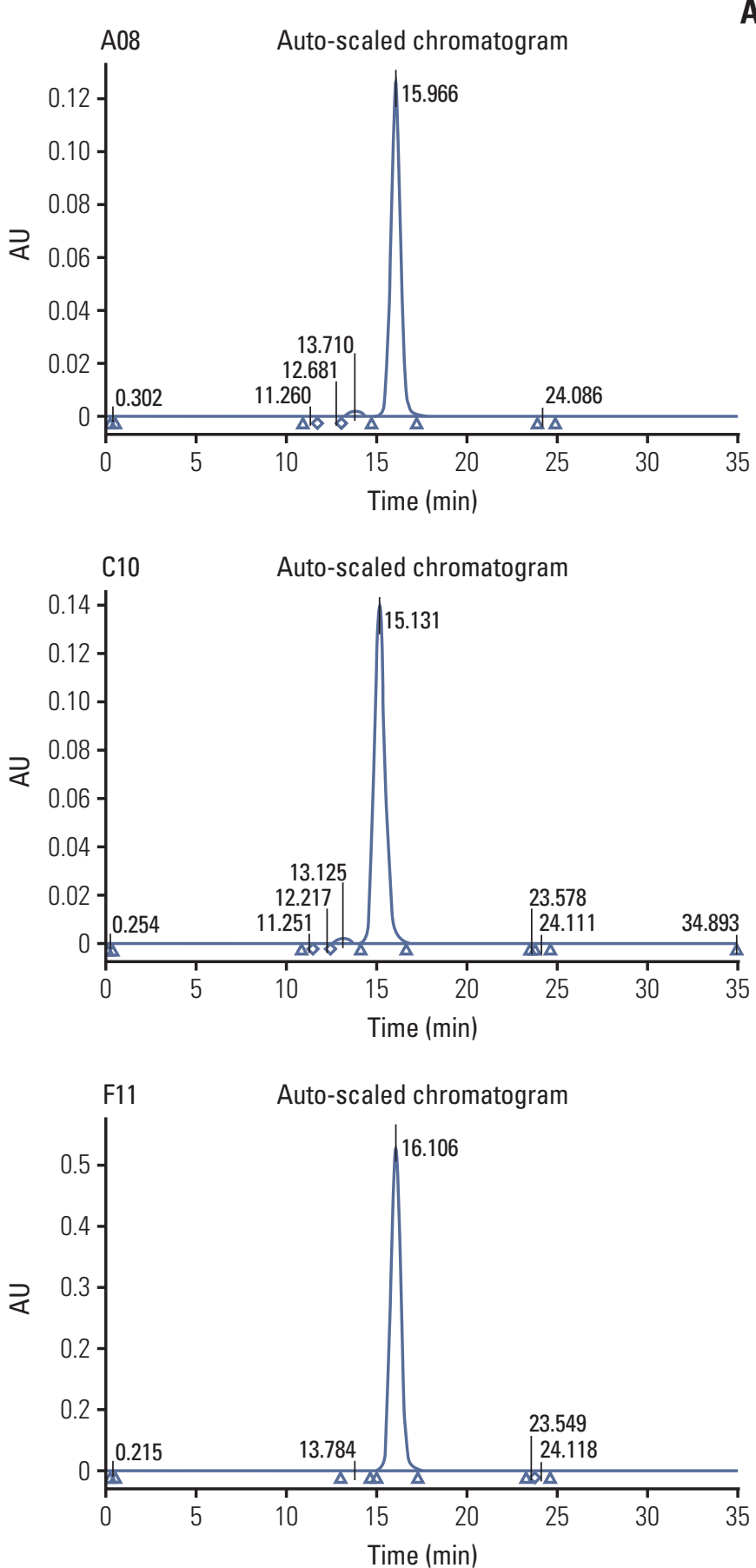

A

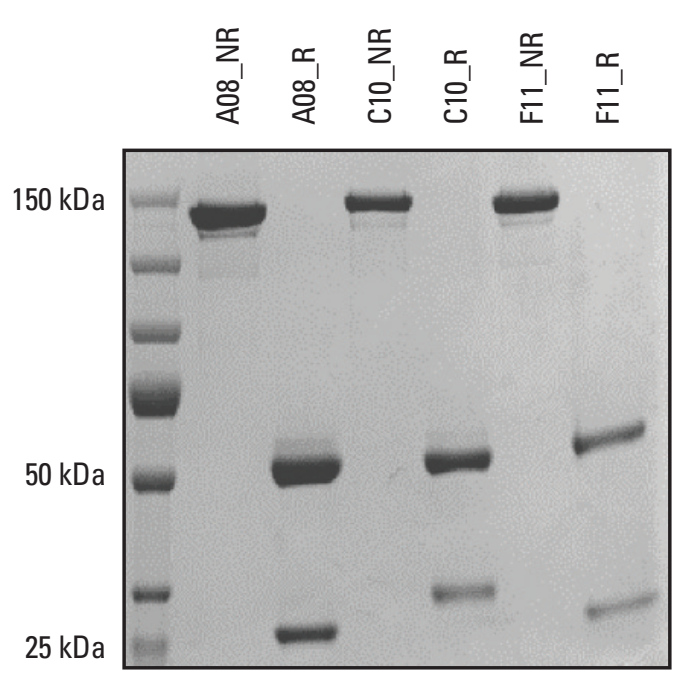

B

C

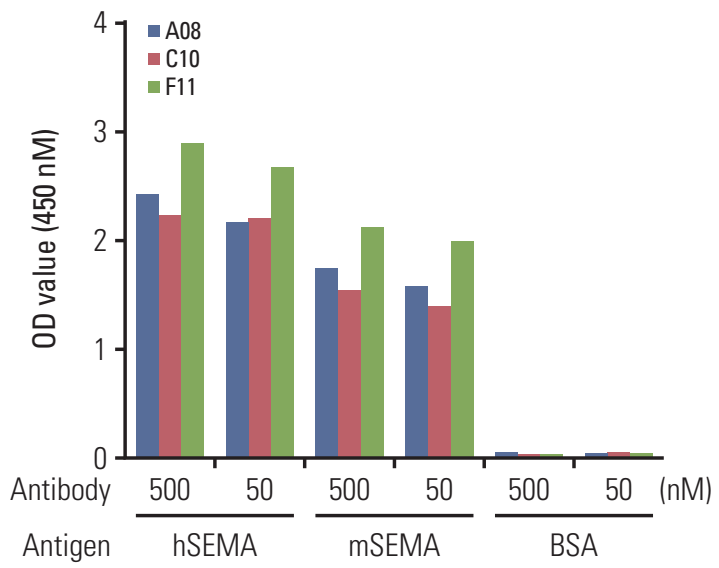

Fig. 3. Production of anti-SEMA3A IgG antibodies. (A) Purity of three anti-SEMA3A IgGs determined by high performance liquid chromatography. The peak at 15-16 minutes indicates $\gamma$-globulin $(\sim 150 \mathrm{kDa})$. The purity of all three anti-SEMA3A IgGs was over 98\%. (B) Sizes of the anti-SEMA3A IgGs were verified in non-reducing (NR) and reducing (R) conditions by sodium dodecyl sulfate polyacrylamide gel electrophoresis. In NR condition, fully IgG was detected whereas in R condition where cleavages disulfide bond heavy chain and light chain were observed. (C) To determinate binding activity of human and mouse SEMA3A, three anti-SEMA3A IgGs were analyzed on enzyme-linked immunosorbent assay (ELISA) plates when the presence of hSEMA3A, mSEMA3A, and bovine serum albumin (BSA) as negative control. Optical density (OD) values derived from indirect ELISA in which SEMA3A IgG was captured via anti-human Fab antibody conjugated to horseradish peroxidase. (Continued to the next page) 

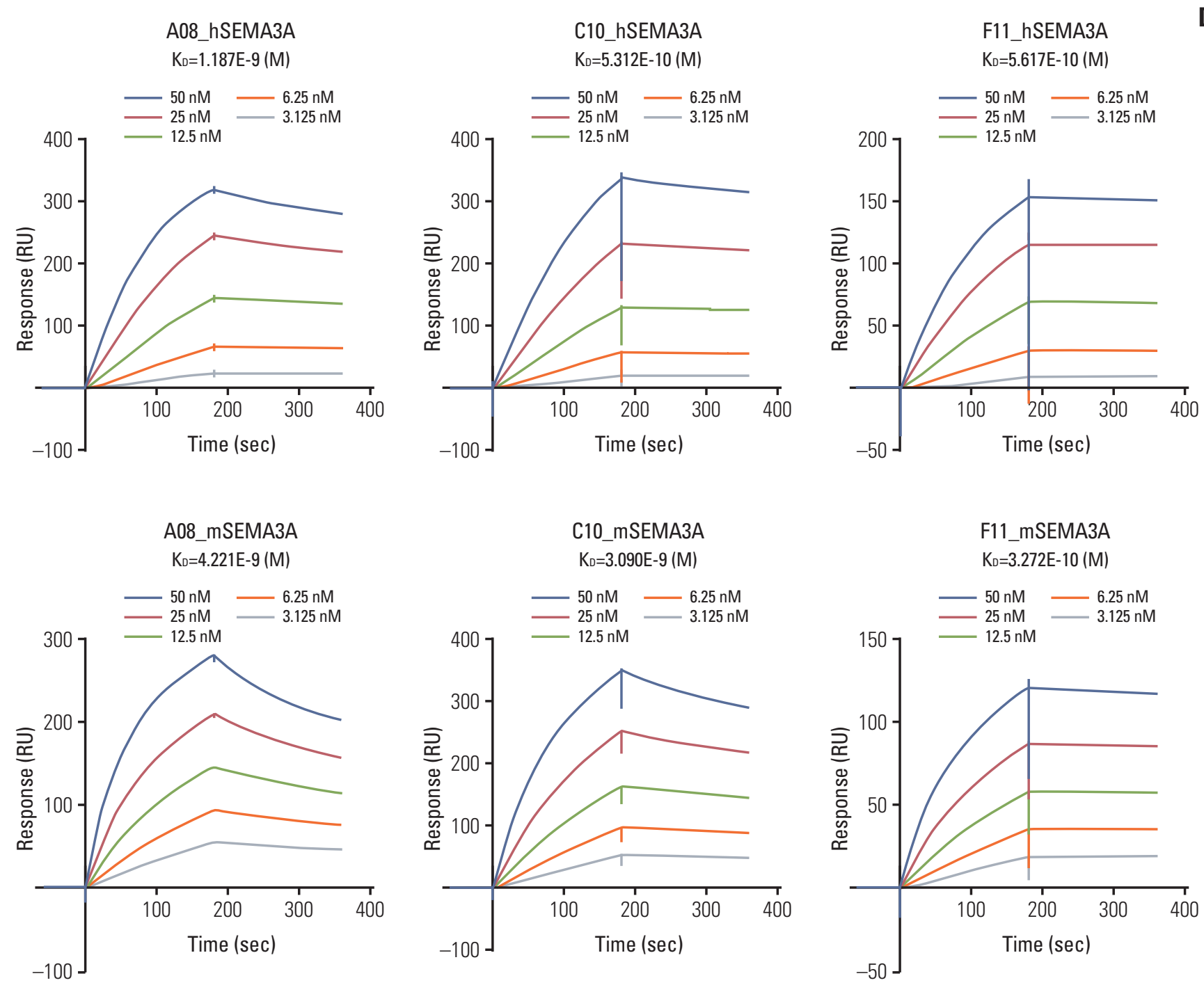

Fig. 3. (Continued from the previous page) (D) Use of the BIAcore to determine the calculated $K_{D}$ value of three anti-SEMA3A IgGs to human and mouse SEMA3A. The assay method was Fab-based capture format via human Fab capture kit (GE Healthcare).

98\%, 98.5\%, and 99\% purity, respectively (Fig. 3A). To validate the physical sizes of the anti-SEMA3A IgG antibodies, we conducted SDS-PAGE analysis under "reducing" and "non-reducing" conditions. Under the non-reducing condition, all three antibodies exhibited a molecular weight of 120 $\mathrm{kDa}$, whereas, under the reducing condition, we observed two major bands at 50 and $25 \mathrm{kDa}$ each, corresponding to the heavy and light chain regions of the antibody. Meanwhile, C10 contained N-glycosylation site in the CDR1 region of the light chain; thus, the molecular size of its light chain was at $30 \mathrm{kDa}, 5 \mathrm{kDa}$ higher than A08 and F11 (Fig. 3B).
To verify the binding activity of the anti-SEMA3A IgG antibodies to both human and mouse SEMA3A, we performed ELISA and surface plasmon resonance assays. As the mouse SEMA3A sequence harbors high homology to the human SEMA3A gene, at approximately 95\%, all three antibodies showed positive cross-reactivity (Fig. 3C). Afterwards, we measured the $\mathrm{K}_{\mathrm{D}}$ (the equilibrium dissociation constant between the antibody and its antigen) values of all three SEMA3A antibodies and discovered that the F11 IgG antibody possessed the highest binding affinity capability to both human and mouse SEMA3A, consistent with the ELISA 

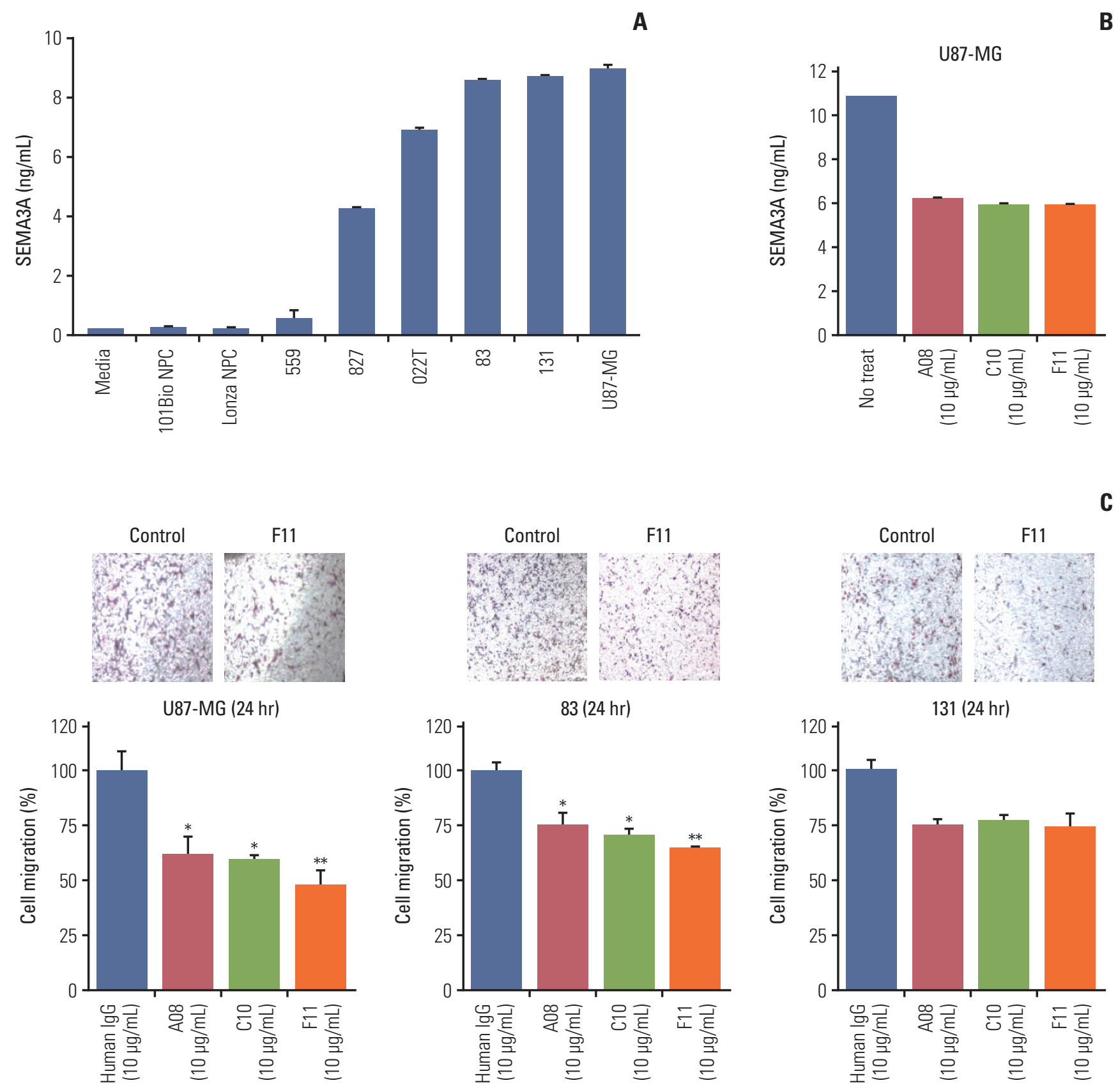

Fig. 4. Anti-SEMA3A IgG antibody impedes migration and proliferation of patient-derived glioblastoma (GBM) cells. (A) The concentration of SEMA3A secreted for a day was measured by sandwich enzyme-linked immunosorbent assay (ELISA) in various cell types. GBM patient cells and U87-MG cells as a positive control and neural progenitor cell and medium as negative controls. (B) Neutralization assay of three anti-SEMA3A IgGs in U87-MG cells. The evaluate method is same method for U87-MG cells were incubated with anti-SEMA3A IgG and the amount of SEMA3A in medium was measured by sandwich ELISA. (C) While the migration rate of cells (U87-MG, 131, and 83) treated with control human IgG remains unchanged, migration rates of cells treated with anti-SEMA3A IgGs for 1 day were impeded significantly. Each antibody treatment concentration was $10 \mu \mathrm{g} / \mathrm{mL}$ and macroscopic observation of the transwell chambers. ${ }^{*} \mathrm{p}<0.05,{ }^{* *} \mathrm{p}<0.01$. (Continued to the next page) 
D

U87-MG (24 hr)

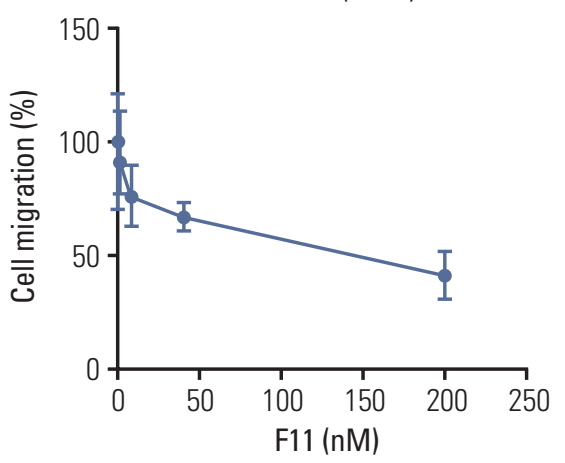

E

131

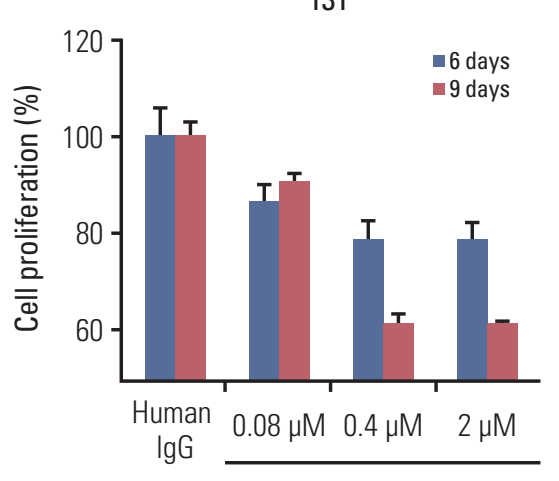

F11

Fig. 4. (Continued from the previous page) (D) Inhibition of migration activity by anti-SEMA3A F11 for one day was evaluated using Oris cell migration assay. The level of migration activity inhibition was proportional to the concentration gradient of anti-SEMA3A F11. Migration activity was reduced by 52\% compared to the control IgG-treated cells in the max concentration of F11 (200 nM) treated cells. (E) Comparison of the effect of anti-SEMA3A F11 and control human IgG (2 $\mu$ M) on in-vitro proliferation of 131 cells. On the 9 days after F11 treatment, 131 proliferation was inhibited by $60 \%$ of control human IgG treated cells. (F) Immunoblots of phospho-ERK and ERK in control human IgG or F11 IgG treated 131 and 559 cells. The SEMA3A hypersecreting 131 cells decreased ERK phosphorylation during F11 treatment and remained unchanged for ERK phosphorylation of 559 cells that secretes less SEMA3A.

data (Fig. 3D). Collectively, our results demonstrate successful production of engineered anti-SEMA3A IgG antibodies that are specific to both human and mouse SEMA3A.

\section{Anti-SEMA3A IgG antibody impedes migration and pro- liferation of patient-derived GBM cells}

Previous reports have shown that GBM cells endogenously secrete SEMA3A and that RNA interference-mediated down regulation of SEMA3A was able to inhibit migratory ability of U87-MG cells [18]. To determine whether patient-derived GBM cells secret SEMA3A, we performed sandwich ELISAs with various PDCs, 131, 83, 022, 827, and 559, and U87-MG cell-line and neural progenitor cells (NPC) as positive and negative controls. Patient-derived GBM cells were cultured in NBA neurosphere culture conditions with additional growth factors (epidermal growth factor and basic fibroblast growth factor) and harvested and examined for secretion of SEMA3A. Both 131 and 83 cells secreted SEMA3A at a similar level to that of U87-MG cells, while the secretion level of SEMA3A was minimal in 559 tumor cells (Fig. 4A). Considering the neutralizing activity of our antibodies against SEMA3A, we performed a sandwich ELISA in the presence of anti-SEMA3A antibodies. Notably, all three antibodies were able to neutralize SEMA3A in U87-MG cells at 10 $\mu \mathrm{g} / \mathrm{mL}$.

Based on their SEMA3A neutralizing effects, we per- formed migration assays to determine the invasive inhibitory effect of anti-SEMA3A IgG antibodies. GBM cells were incubated in presence of different anti-SEMA3A IgG antibodies or control human $\operatorname{IgG}(10 \mu \mathrm{g} / \mathrm{mL})$. Interestingly, all three antibodies showed significant migration suppression in U87MG, 83, and 131 cells (Fig. 4C). As expected, the F11 antibody, which showed the highest $\mathrm{K}_{\mathrm{D}}$ value, exhibited greater inhibitory effects compared to the other antibodies. Using the Oris cell migration assay, we observed a dose-dependent migration inhibition of U87-MG cells in the presence of F11 antibody. (Fig. 4D). We then determined whether the proliferative response to anti-SEMA3A F11 was applicable in patient-derived GBM cells using Ez-Cytox cell viability assay. Under SEMA3A scFvs treatment, the cell proliferation rates were dependent on the secretion levels of SEMA3A from the GBM PDCs (S2 Fig.). Our results showed that the treatment with F11 antibody exhibited a dramatic reduction in cellular proliferation compared to the human IgG (Fig. 4E). Previous studies have shown that MAPK pathway including ERK1/2 induces cellular proliferation in gliomas [25]. Notably, immunoblot assay revealed that phospho-ERK level was significantly attenuated in 131 GBM cells that were pre-incubated with F11 for 30 minutes, whereas no notable changes were detected from the 559 cells (Fig. 4F). These results demonstrated that the F11 anti-SEMA3A IgG antibody effectively inhibited SEMA3A-induced migration and proliferation in GBM. 
A
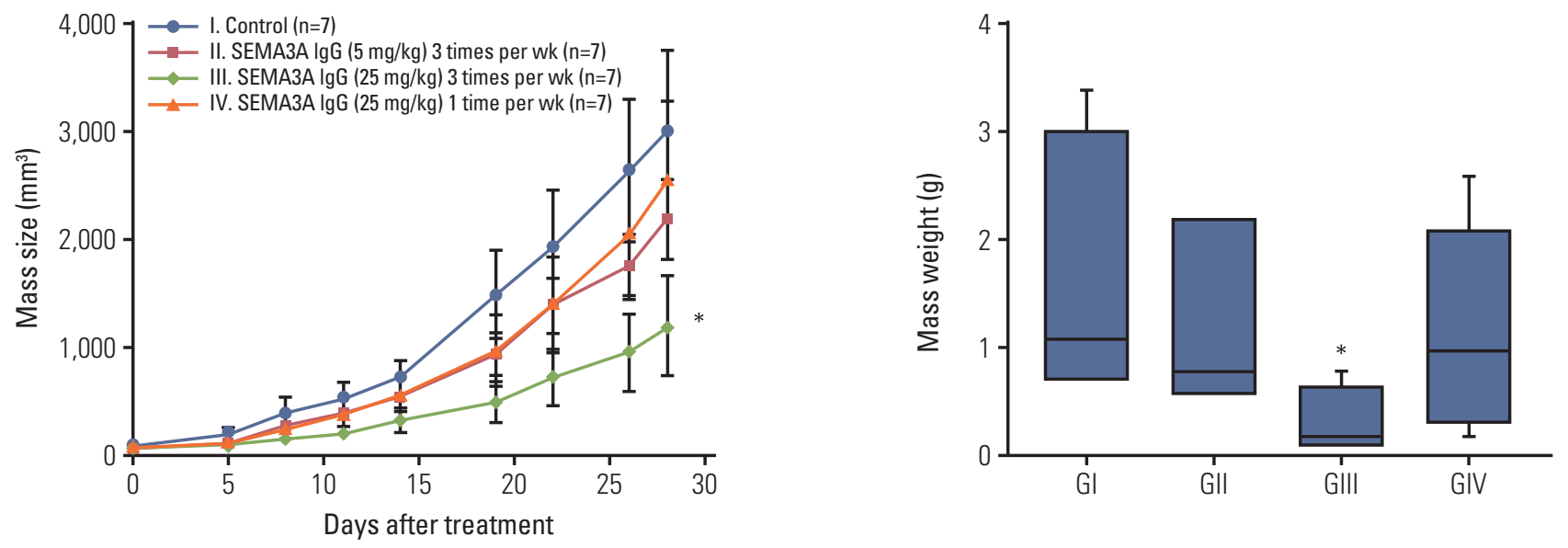

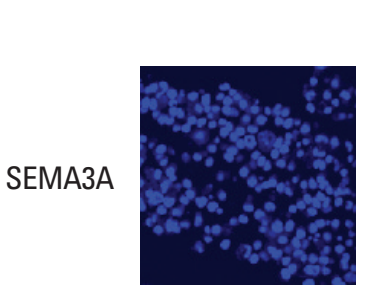

Control
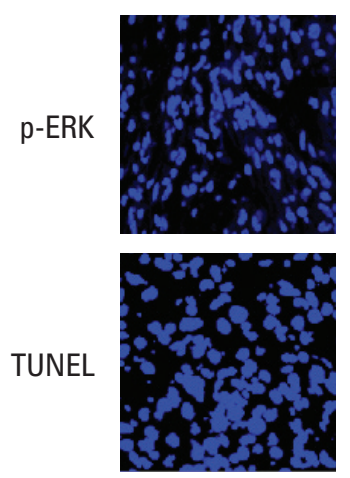
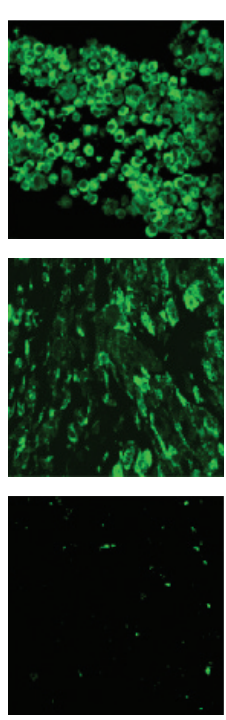
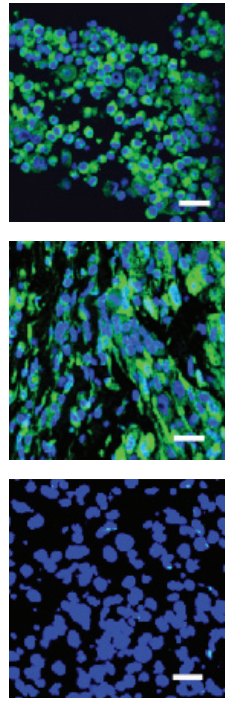

Anti-SEMA3A
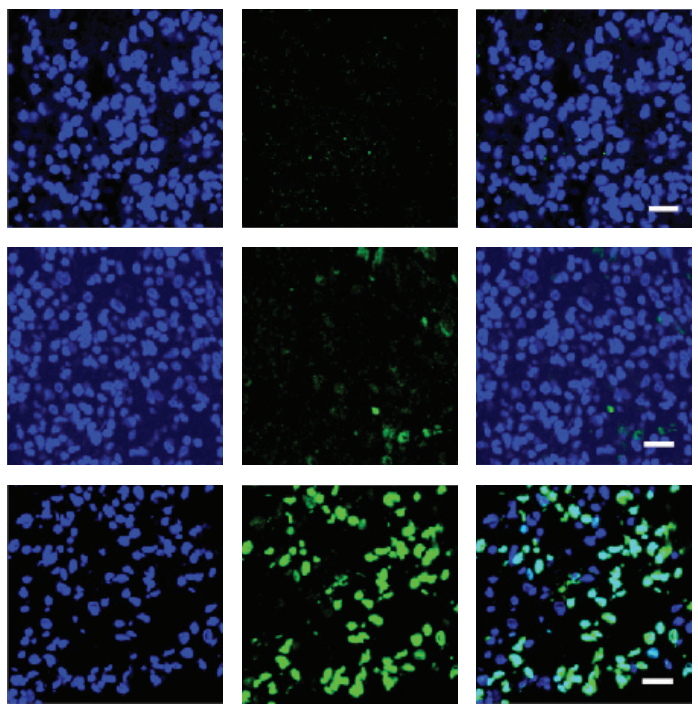

Fig. 5. In vivo effects of anti-SEMA3A antibody on the glioblastoma (GBM) tumor growth. (A) Antitumor activity of antiSEMA3A F11 in GBM patient cell 131 xenograft model. When tumors were approximately $80 \mathrm{~mm}^{3}$, the patient-derived xenograft tumors were randomly assigned into study groups ( $\mathrm{n}=7 \mathrm{mice} /$ group) and were participated in the dosing phase of the study. Treatment of F11 was administered intravenously at $5 \mathrm{mg} / \mathrm{kg}$ and $25 \mathrm{mg} / \mathrm{kg}$ with all treatments well tolerated and anti-SEMA3A F11 injections were stopped at day 28. ${ }^{*} \mathrm{p}<0.05$. (B) Mass weight variation of mice was similar to mass size affected by anti-SEMA3A F11 within groups. ${ }^{*} p<0.05$. (C, D) Representative immunofluorescence images of SEMA3A, p-ERK, terminal deoxynucleotidyl transferase dUTP nick end labeling (TUNEL) and Iba1 staining using paraffin sections of tumors. Scale bars=20 $\mu \mathrm{m}$ (white), $100 \mu \mathrm{m}$ (red). (Continued to the next page)

\section{In vivo effects of anti-SEMA3A antibody on GBM tumor growth}

Based on in vitro migratory and proliferative inhibition effect of the F11 antibody, we further evaluated its efficacy in vivo setting. 131 tumor xenograft models were generated and treated with either PBS or F11 via intravenous injection. As suspected, treatment of tumor-bearing mice with 25 $\mathrm{mg} / \mathrm{kg}$ of F11 antibody decreased the tumor growth by $60 \%$ compared to the PBS-treated group (Fig. 5A). Our results 

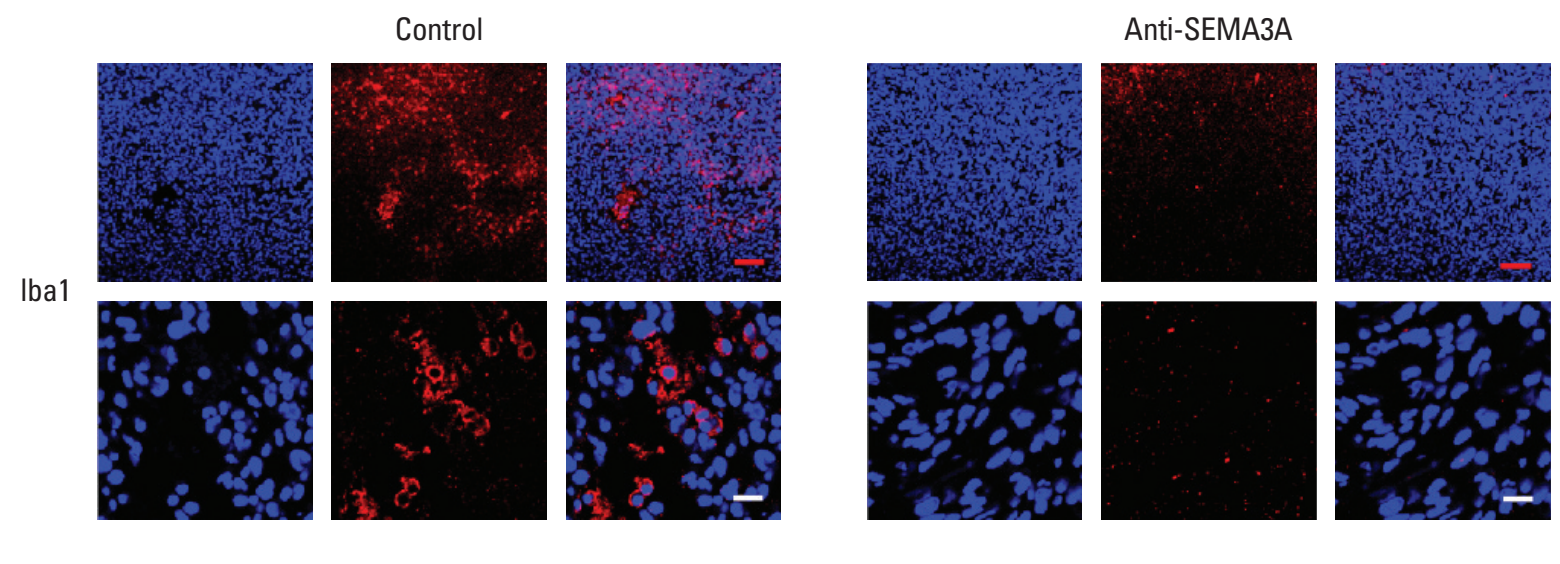

Fig. 5. (Continued from the previous page)

were consistently observed through tumor weight analysis in the corresponding mice (Fig. 5B). Mice that were treated with either F11 antibody or control showed no significant weight loss or neurological symptoms in S3 Fig.

Immunofluorescence staining of F11 antibody-treated tumors demonstrated significant reduction of SEMA3A and phosphor-ERK compared to the control tumor (Fig. 5C). Additionally, TUNEL assay showed F11 antibody-induced apoptotic cells, suggesting that F11 treatment can induce apoptotic cell death in vivo (Fig. 5C).

Previous studies have shown dynamic interaction between SEMA3A and tumor microenvironment. SEMA3A/NRP1 signaling promotes TAM infiltration and pro-tumorigenic function in hypoxic tumor regions [26,27]. Therefore, we interrogated the potential effect of SEMA3A neutralization on tumor microenvironment. Interestingly, TAMs were extensively recruited by adjacent tumor cells secreting SEMA3A in the control tumors, while significantly less accumulated TAM population was observed in the F11treated tumors (Fig. 5D). Collectively, our results demonstrate that SEMA3A inhibitor could prevent tumor proliferation and TAM infiltration in vivo, highlighting antiSEMA3A F11 antibody as a potential therapeutic agent in GBM therapy.

\section{Discussion}

SEMA3A is highly expressed in GBM compared to nonneoplastic brain tissues and SEMA3A signaling axis promotes GBM growth and invasion, making it an ideal therapeutic target in a clinical framework. Nevertheless, previous studies only showed RNA interference-mediated SEMA3A disruption that lead to migration inhibition [18]. Although siRNA has been widely used as an effective tool to down-regulate target genes, it cannot be translated into a clinical course. Therefore, we evaluated SEMA3A antibody as a potential therapeutic agent against GBM progression.

In present study, we have developed single-chain antibodies that are specific to Semaphorin3A from synthetic phage library using a classical panning method. To examine whether primary GBM cells secret SEMA3A, we used sandwich ELISA assay and compared the secretion level of SEMA3A in patient-derived GBM cells relative to NPC and U87-MG cells. We then converted the generated SEMA3A scFvs into three fully human IgG forms. IgG transformation not only increased their binding affinities, but also maintained their functional roles in cellular migration and proliferation inhibition. As the produced anti-SEMA3A antibodies are fully human, they may serve as a safer therapeutic agent in human disease. Furthermore, all three IgG antibodies showed specific cross-reactivity both mouse and human SEMA3A, allowing them to bypass in vivo immune response as well.

Previous studies have shown that SEMA3A modulates cell dispersal via activating multiple signaling pathways including Rac1, GSK3b, and ERK1/2 in various cancer cells. All three anti-SEMA3A antibodies were shown to repress migration of patient-derived GBM and U87-MG cell line. Furthermore, we confirmed the neutralization effect of antiSEMA3A antibody using U87-MG cell line.

Dynamic interactions between tumor cells and tumor microenvironment frequently constitute tumor malignancy [28]. According to recent studies, TAMs are recruited to avas- 
cular areas in hypoxic tumor environment, resulting in sustained tumor progression [27]. SEMA3A acts as a chemoattractant by TAMs via VEGFR1 phophorylation and recruits TAMs towards hypoxic niches in NRP1-independent manner [26]. We confirmed that these results corroborate with our in vivo study demonstrating that anti-SEMA3A IgG suppresses tumor growth dramatically through preventing TAM recruitment.

Given that extracellular vesicle-derived SEMA3A as a propermeability factor that induces loss of barrier integrity in GBM, it could potentially be integrated for prognostic purposes. Our study also presents anti-SEMA3A IgG as a candidate diagnostic tool in monitoring GBM [19]. Recently, SEMA3A was evaluated as therapeutic target in ischemic or diabetic retinopathy, neurotrophic corneal disease, spinal cord injury and tumor progression [29]. Therefore, antiSEMA3A antibody could be employed as a new diagnostic and therapeutic agent for GBM patients and other diseases that are related to SEMA3A.

\section{Electronic Supplementary Material}

Supplementary materials are available at Cancer Research and Treatment website (http://www.e-crt.org).

\section{Conflicts of Interest}

Conflict of interest relevant to this article was not reported.

\section{Acknowledgments}

This research was supported by a grant of the Korea Health Technology R\&D Project through the Korea Health Industry Development Institute (KHIDI), funded by the Ministry of Health \& Welfare, Republic of Korea. (HI14C3418). Additional supports were from NIH R01 NS082312 and R01 NS 083767 (JL).

\section{References}

1. Adamson C, Kanu OO, Mehta AI, Di C, Lin N, Mattox AK, et al. Glioblastoma multiforme: a review of where we have been and where we are going. Expert Opin Investig Drugs. 2009;18:1061-83.

2. Louis DN, Ohgaki H, Wiestler OD, Cavenee WK, Burger PC, Jouvet A, et al. The 2007 WHO classification of tumours of the central nervous system. Acta Neuropathol. 2007;114:97-109.

3. Reardon DA, Wen PY. Therapeutic advances in the treatment of glioblastoma: rationale and potential role of targeted agents. Oncologist. 2006;11:152-64.

4. Stupp R, Mason WP, van den Bent MJ, Weller M, Fisher B, Taphoorn MJ, et al. Radiotherapy plus concomitant and adjuvant temozolomide for glioblastoma. N Engl J Med. 2005;352: 987-96.

5. Linger RM, Keating AK, Earp HS, Graham DK. TAM receptor tyrosine kinases: biologic functions, signaling, and potential therapeutic targeting in human cancer. Adv Cancer Res. 2008;100:35-83.

6. Hanahan D, Weinberg RA. Hallmarks of cancer: the next generation. Cell. 2011;144:646-74.

7. Dunn GP, Rinne ML, Wykosky J, Genovese G, Quayle SN, Dunn IF, et al. Emerging insights into the molecular and cellular basis of glioblastoma. Genes Dev. 2012;26:756-84.

8. Zwick E, Bange J, Ullrich A. Receptor tyrosine kinase signalling as a target for cancer intervention strategies. Endocr Relat Cancer. 2001;8:161-73.

9. Herbst RS, Shin DM. Monoclonal antibodies to target epider- mal growth factor receptor-positive tumors: a new paradigm for cancer therapy. Cancer. 2002;94:1593-611.

10. Sliwkowski MX, Mellman I. Antibody therapeutics in cancer. Science. 2013;341:1192-8.

11. Tamagnone L, Comoglio PM. Signalling by semaphorin receptors: cell guidance and beyond. Trends Cell Biol. 2000;10: 377-83.

12. Kruger RP, Aurandt J, Guan KL. Semaphorins command cells to move. Nat Rev Mol Cell Biol. 2005;6:789-800.

13. Neufeld G, Sabag AD, Rabinovicz N, Kessler O. Semaphorins in angiogenesis and tumor progression. Cold Spring Harb Perspect Med. 2012;2:a006718.

14. Luo Y, Raible D, Raper JA. Collapsin: a protein in brain that induces the collapse and paralysis of neuronal growth cones. Cell. 1993;75:217-27.

15. Kigel B, Varshavsky A, Kessler O, Neufeld G. Successful inhibition of tumor development by specific class-3 semaphorins is associated with expression of appropriate semaphorin receptors by tumor cells. PLoS One. 2008;3:e3287.

16. Acevedo LM, Barillas S, Weis SM, Gothert JR, Cheresh DA. Semaphorin 3A suppresses VEGF-mediated angiogenesis yet acts as a vascular permeability factor. Blood. 2008;111:2674-80.

17. Muller MW, Giese NA, Swiercz JM, Ceyhan GO, Esposito I, Hinz U, et al. Association of axon guidance factor semaphorin $3 \mathrm{~A}$ with poor outcome in pancreatic cancer. Int J Cancer. 2007;121:2421-33.

18. Bagci T, Wu JK, Pfannl R, Ilag LL, Jay DG. Autocrine sema- 
phorin 3A signaling promotes glioblastoma dispersal. Oncogene. 2009;28:3537-50.

19. Treps L, Edmond S, Harford-Wright E, Galan-Moya EM, Schmitt A, Azzi S, et al. Extracellular vesicle-transported Semaphorin3A promotes vascular permeability in glioblastoma. Oncogene. 2016;35:2615-23.

20. Hu ZQ, Zhou SL, Zhou ZJ, Luo CB, Chen EB, Zhan H, et al. Overexpression of semaphorin $3 \mathrm{~A}$ promotes tumor progression and predicts poor prognosis in hepatocellular carcinoma after curative resection. Oncotarget. 2016;7:51733-46.

21. Kretzschmar T, von Ruden T. Antibody discovery: phage display. Curr Opin Biotechnol. 2002;13:598-602.

22. Hoogenboom HR, de Bruine AP, Hufton SE, Hoet RM, Arends JW, Roovers RC. Antibody phage display technology and its applications. Immunotechnology. 1998;4:1-20.

23. Lee J, Kotliarova S, Kotliarov Y, Li A, Su Q, Donin NM, et al. Tumor stem cells derived from glioblastomas cultured in bFGF and EGF more closely mirror the phenotype and genotype of primary tumors than do serum-cultured cell lines. Cancer Cell. 2006;9:391-403.

24. Yang HY, Kang KJ, Chung JE, Shim H. Construction of a large synthetic human scFv library with six diversified CDRs and high functional diversity. Mol Cells. 2009;27:225-35.

25. Shinohara H, Yagita H, Ikawa Y, Oyaizu N. Fas drives cell cycle progression in glioma cells via extracellular signal-regulated kinase activation. Cancer Res. 2000;60:1766-72.

26. Casazza A, Laoui D, Wenes M, Rizzolio S, Bassani N, Mambretti $\mathrm{M}$, et al. Impeding macrophage entry into hypoxic tumor areas by Sema3A/Nrp1 signaling blockade inhibits angiogenesis and restores antitumor immunity. Cancer Cell. 2013;24:695-709.

27. Rivera LB, Bergers G. Location, location, location: macrophage positioning within tumors determines pro- or antitumor activity. Cancer Cell. 2013;24:687-9.

28. Solinas G, Germano G, Mantovani A, Allavena P. Tumorassociated macrophages (TAM) as major players of the cancer-related inflammation. J Leukoc Biol. 2009;86:1065-73.

29. Cerani A, Tetreault N, Menard C, Lapalme E, Patel C, Sitaras $\mathrm{N}$, et al. Neuron-derived semaphorin $3 \mathrm{~A}$ is an early inducer of vascular permeability in diabetic retinopathy via neuropilin-1. Cell Metab. 2013;18:505-18. 